\title{
Controlling inbreeding and maximizing genetic gain using semi-definite programming with pedigree-based and genomic relationships
}

\author{
S. Schierenbeck, ${ }^{\star} \dagger^{1}$ E. C. G. Pimentel,‡ M. Tietze, ${ }^{\star} \ddagger$ J. Körte,§ R. Reents, $†$ F. Reinhardt, $†$ H. Simianer, ${ }^{*}$ \\ and S. Königł \\ *Animal Breeding and Genetics Group, Department of Animal Sciences, Georg-August-University of Göttingen, D-37075 Göttingen, Germany \\ †Vereinigte Informationssysteme Tierhaltung w.V., D-27283 Verden, Germany \\ ‡Department of Animal Breeding, University of Kassel, D-37213 Witzenhausen, Germany \\ §Institute for Animal Breeding and Husbandry, Christian-Albrechts-University of Kiel, D-24118 Kiel
}

\section{ABSTRACT}

Because of the relatively high levels of genetic relationships among potential bull sires and bull dams, innovative selection tools should consider both genetic gain and genetic relationships in a long-term perspective. Optimum genetic contribution theory using official estimated breeding values for a moderately heritable trait (production index, Index-PROD), and a lowly heritable functional trait (index for somatic cell score, Index-SCS) was applied to find optimal allocations of bull dams and bull sires. In contrast to previous practical applications using optimizations based on Lagrange multipliers, we focused on semi-definite programming (SDP). The SDP methodology was combined with either pedigree $\left(a_{i j}\right)$ or genomic relationships $\left(f_{i j}\right)$ among selection candidates. Selection candidates were 484 genotyped bulls, and 499 preselected genotyped bull dams completing a central test on station. In different scenarios separately for PROD and SCS, constraints on the average pedigree relationships among future progeny were varied from $a_{i j}=0.08$ to $a_{i j}=0.20$ in increments of 0.01. Corresponding constraints for single nucleotide polymorphism-based kinship coefficients were derived from regression analysis. Applying the coefficient of 0.52 with an intercept of 0.14 estimated for the regression pedigree relationship on genomic relationship, the corresponding range to alter genomic relationships varied from $f_{i j}=0.18$ to $f_{i j}=0.24$. Despite differences for some bulls in genomic and pedigree relationships, the same trends were observed for constraints on pedigree and corresponding genomic relationships regarding results in genetic gain and achieved coefficients of relationships. Generally, allowing higher values for relationships resulted in an increase of genetic gain for Index-PROD and Index-SCS and in a reduction in the number of

Received May 26, 2011.

Accepted July 22, 2011.

${ }^{1}$ Corresponding author: sven.schierenbeck@vit.de selected sires. Interestingly, more sires were selected for all scenarios when restricting genomic relationships compared with restricting pedigree relationships. For example, at constraint of $f_{i j}=0.185$ and selection on Index-PROD, the number of selected sires was 35 . In contrast, only 21 sires were selected at the comparable constraint on additive genetic relationship of $\mathrm{a}_{i j}=0.09$. A further reduction in relationships is possible when using SDP output (i.e., suggested genetic contributions of selected parents) and applying a simulated annealing algorithm to define specific mating plans. However, the advantage of this strategy is limited to a short-term perspective and probably not successful in the period of genomic selection allowing a substantial reduction of generation intervals.

Key words: inbreeding, optimum genetic contribution, pedigree and genomic relationship

\section{INTRODUCTION}

The methodology of genomic selection enables the estimation of genomic breeding values of young sires with relatively high accuracy for production as well as for functional traits (e.g., VanRaden et al., 2009). An expected consequence is a further increase of selection intensities in genomic breeding programs on the cow sire and bull sire pathways of selection. König et al. (2009) economically evaluated genomic breeding programs based on the general framework of a genomic breeding program design as developed by Schaeffer (2006). Gain in discounted profit was strongly correlated with the reduction in the number of young sires having been selected upon genomic breeding values. For example, when selecting 25 young sires per year to inseminate a population of 50,000 cows, discounted profit per cow and year was €50. Profit was doubled when increasing selection intensity and selecting only 5 outstanding young sires. Hence, to balance genetic gain and inbreeding in a long-term perspective in the genomic era, König et al. (2009) suggested the use of 
genomic information (i.e., SNP pattern and genomic EBV) and to focus on both aspects - selection response and constraints in relationships - among selection candidates.

The upward trend of inbreeding level in different dairy cattle populations due (mainly) to the widespread distribution of sire semen has been evaluated in several studies in the past $20 \mathrm{yr}$ (e.g., Wiggans et al., 1995; Miglior, 2000; Thompson et al., 2000; Kearney et al., 2004). Because of the effect of selection on phenotypic means, genetic variances, and changes in relationships of individuals to the population, VanRaden (2005) described a method for adjusting expected future inbreeding that can be included in the process of genetic evaluation. According to VanRaden (2005), this adjustment procedure was introduced in several US trait evaluations in February 2005. An approach used by König and Simianer (2006) to maximize genetic gain by restricting additive genetic relationships among bull sires and bull dams was based on quantitative genetics and optimum genetic contributions (OGC) as developed in theory by Meuwissen (1997). König and Simianer (2006) strongly recommended controlling inbreeding when mating bull sires to bull dams, because both genetic groups have a substantial effect on the development of genetic relationships in dairy cattle breeding programs. Available mating programs used by breeding organizations or dairy cattle producers suggest cow sires for matings with cow dams. Such a mating strategy implies the management of inbreeding and relationships only in the short term. In contrast, long-term control of inbreeding in a dairy population requires consideration of relationships between young bulls entering AI progeny test programs, or directly selected sires for AI based on genomic breeding values. The program GENCONT (Meuwissen, 2002) was used by König and Simianer (2006) for elite matings in a breeding program embracing 30 young bulls per year to find the optimal allocations of bull sires and bull dams for one specific breeding organization. Compared with the actual breeding program applied in practice, OGC theory revealed the potential to increase genetic gain under the same constraint for the increase of average relationship by $13.1 \%$.

Algorithms as implemented in GENCONT (Meuwissen, 2002) are based upon a series of relaxed optimizations using Lagrange multipliers. This methodology has been intensively discussed by Pong-Wong and Woolliams (2007), and they focused on the problem that invalid negative genetic contributions could be assigned to some individuals. As an approximate solution, GENCONT fixes genetic contributions of those individuals to zero, and the algorithm continues in a smaller subset of selection candidates. An alternative suggested by Pong-Wong and Woolliams (2007) for maximizing genetic gain while restricting inbreeding is a method based upon semi-definite programming (SDP). Due to the failure of Lagrange multipliers in some situations; that is, the possible negative contributions when applying GENCONT, expected gains with SDP for some examples were substantially higher, in the range from 1.5 to $9 \%$.

Practical applications of OGC theory in dairy cattle are mainly based on GENCONT and use additive genetic relationship matrices among selection candidates (e.g., Kearney et al., 2004; König and Simianer, 2006). Additive genetic relationship matrices use only pedigree data to calculate probabilities that gene pairs are identical by descent (Wright, 1922). However, wrong and missing sire information for Holstein dairy cattle in the assumed range from 3 to $23 \%$ (e.g., Weller et al., 2004) is a well-known problem for setting up accurate additive genetic relationships. Further problems arise when applying BLUP animal or BLUP sire models (i.e., possibly biased EBV), especially for low heritability traits. Paternity errors are due to recording errors by the farmer or by the AI company (Sanders et al., 2006). Alternatively, the use of actual SNP genotypes for construction of genomic relationship matrices can provide more accurate measures of realized relationships among animals. Pimentel et al. (2011a) estimated the association of kinship coefficients from marker data against relationship coefficients estimated from pedigree data for genotyped bulls in Germany; the $\mathrm{R}^{2}$ value was only 0.53 . Accordingly, the authors suggested the use of genomic relationships for further association analyses due to the potential sire and dam misidentification when using pedigree relationships. Furthermore, as pointed out by Schork (2001), relationship coefficients calculated from pedigree data do not take into account the variation in relationships among similarly related individuals. Such variation is accounted for when computing relationship coefficients using marker information. Consequently, the aim of our study was to generate a framework for the combination of SDP methodology with genomic relationships among selection candidates, and to determine optimal genetic contributions for elite mating schemes for the optimization of genomic breeding programs. When restricting selection of candidates according to genomic relationships, realized additive genetic relationships were monitored, and vice versa.

\section{MATERIALS AND METHODS}

\section{Data}

The data file of selection candidates consisted of 484 German Holstein bulls born in 2002 that were part of 
Table 1. Means, standard deviation (SD), minimum and maximum for the selection criteria production index (Index-PROD) and SCS index (Index-SCS) for bull dams and bull sires

\begin{tabular}{llllccc}
\hline & & \multicolumn{4}{c}{ Descriptive statistic } \\
\cline { 3 - 6 } Group & Number & Index & Mean & SD & Minimum & Maximum \\
\hline Bull dams & \multirow{2}{*}{499} & PROD & 103.27 & 6.61 & 81 & 121 \\
\multirow{2}{*}{ Bull sires } & & SCS & 103.24 & 8.60 & 79 & 126 \\
& \multirow{2}{*}{484} & PROD & 103.15 & 12.26 & 65 & 141 \\
& & SCS & 102.23 & 11.15 & 67 & 136 \\
\hline
\end{tabular}

the German reference population for the estimation of SNP effects. Thus, their EBV were very similar to their genomic breeding values. All bulls were included without prior selection on EBV. Furthermore, 499 preselected potential bull dams born from 2001 to 2006 and located at a central test station were included in the data set. All selection candidates had been genotyped, and they had an official national EBV for the overall production index (Index-PROD), and for the somatic cell score index (Index-SCS). Means and standard deviations for both indices are shown in Table 1.

\section{Methods}

Coefficients for Relationship Matrices. Coefficients of kinship among genotyped bull sires and bull dams were calculated following the similarity index approach proposed by Eding and Meuwissen (2001). For each SNP locus, a genetic similarity index between a pair of bulls $x$ and $y$ was computed as $S_{x y}=0.25\left(\mathrm{I}_{11}+\right.$ $\mathrm{I}_{12}+\mathrm{I}_{21}+\mathrm{I}_{22}$ ), where $\mathrm{I}_{i j}$ is 1 if allele $i$ in $x$ is identical to allele $j$ in $y$, or 0 otherwise. Under the assumption of unique founder alleles, $S_{x y}$ averaged over multiple loci is an unbiased estimator of the kinship coefficient (i.e., the probability of identical by descent). When founder alleles are not unique, it needs to be corrected for the probability of alleles being alike in state. For a given locus, the kinship coefficient between a pair of animals $i$ and $j\left(f_{i j}\right)$ was therefore estimated as

$$
\hat{f}_{i j}=\frac{S_{i j}-s}{1-s},
$$

where $s$ is the similarity index in the founder population; that is, the probability of 2 alleles being alike in state but not identical by descent. An estimate of $s$ can be obtained from data on the founder population as follows:

$$
s=\sum q_{k}^{2},
$$

where $q_{k}$ is the frequency of the $k$ th allele of the given locus in the founder population.
Allele frequencies in the founder population were estimated following the mixed model equations method proposed by Gengler et al. (2007) using a pedigree comprising 21,646 animals tracing back to 1906 . Final estimates of kinship coefficients between every pair of animals were computed by averaging across all loci. Since estimates of s differed from one locus to another, the inverse of the variance of the estimate was used as weight when taking the average, as proposed by Eding and Meuwissen (2001). Pedigree-based relationships $a_{i j}$ were calculated by the tabular method (Emik and Terrill, 1949).

SDP Algorithm. Based on the SDP theory for maximizing genetic gain and minimizing long-term inbreeding or relationships, as introduced by Pong-Wong and Woolliams (2007), we developed our own computer program. The flexibility of this computer program allows the use of either pedigree-based relationships or kinship coefficients derived from SNP data as an input file. The basic idea of the SDP algorithm originates from Fujisawa et al. (2002). Accounting for minimum contributions was done in an iterative way by fixing the maximum contribution of the animals with the lowest contributions to zero, and then starting the SDP algorithm again. This was done until all animals exceeded the minimum contribution.

The parameter file for the SDP algorithm to describe the outline of the breeding program was set up in the standard SDP form as described by Pong-Wong and Woolliams (2007). Minimum genetic contributions for bull sires were fixed at $2 \%$, and maximum genetic contributions for individual sires were restricted to $20 \%$, resulting in 10 possible matings. Genetic contributions for bull dams were generally fixed at $2 \%$. This implies an annually generated number of 50 young bulls, assuming that reproduction biotechnologies ensure at least one male offspring per cow and year. This is a realistic success rate of embryo transfer as used by Wensch-Dorendorf et al. (2011) to evaluate genomic dairy cattle breeding programs in Germany via simulation studies.

In different scenarios for Index-PROD and IndexSCS separately, constraints on the average pedigree relationships among future progeny were varied from $a_{i j}$ 
$=0.08$ to $a_{i j}=0.20$ in increments of 0.01 . Corresponding constraints for SNP-based kinship coefficients were derived from regression analysis. Using the regression coefficient for $b_{f_{i j, a_{i j}}}$ of 0.52 with an intercept of 0.14 produces a corresponding range in genomic relationships from $f_{i j}=0.18$ to $f_{i j}=0.24$ (Figure 1 ).

Specific Mating Design. The SDP output (i.e., suggested genetic contributions for bull sires and bull dams) was used to identify specific matings with the ultimate goal of minimizing the average inbreeding coefficient in the short term in the subsequent generation. For this purpose, a simulated annealing algorithm was applied (Press et al., 1989; Sonesson and Meuwissen, 2000). The essential steps of this algorithm have been summarized by König et al. (2010). Further essential input parameters were all possible relationships between pairs of selected bull dams and selected bull sires. The annealing algorithm was applied to the SDP output from 2 constraints on additive genetic relationships, and from the corresponding 2 constraints on genomic relationships. Constraints were $a_{i j}=0.09$ and $a_{i j}=0.14$ and, accordingly, $f_{i j}=0.185$ and $f_{i j}=0.21$.

\section{RESULTS AND DISCUSSION}

\section{SDP Algorithm}

Average additive genetic relationship among 983 selection candidates was $0.098 \pm 0.040$, and average genomic relationship was $0.189 \pm 0.035$ (Table 2). Average relationships among potential bull dams, among potential bull sires, and among bull dams and bull sires are also shown in Table 2. Additive genetic relationships among the best 4,000 index cows in 2006 originating from Great Britain, Italy, and Ireland were 0.083, 0.087 , and 0.107 , respectively (Mrode et al., 2009), and thus comparable to results from our study.

Average values for both indices of selected animals for different constraints on additive genetic and genomic relationships are shown in Figure 2 (Index-PROD) and in Figure 3 (Index-SCS). An increase in Index-PROD was generally associated with an increase in allowed long-term relationships. This is because a relaxation in constraints for relationships resulted in a lower number of selected bulls (Figure 4). Hence, higher genetic

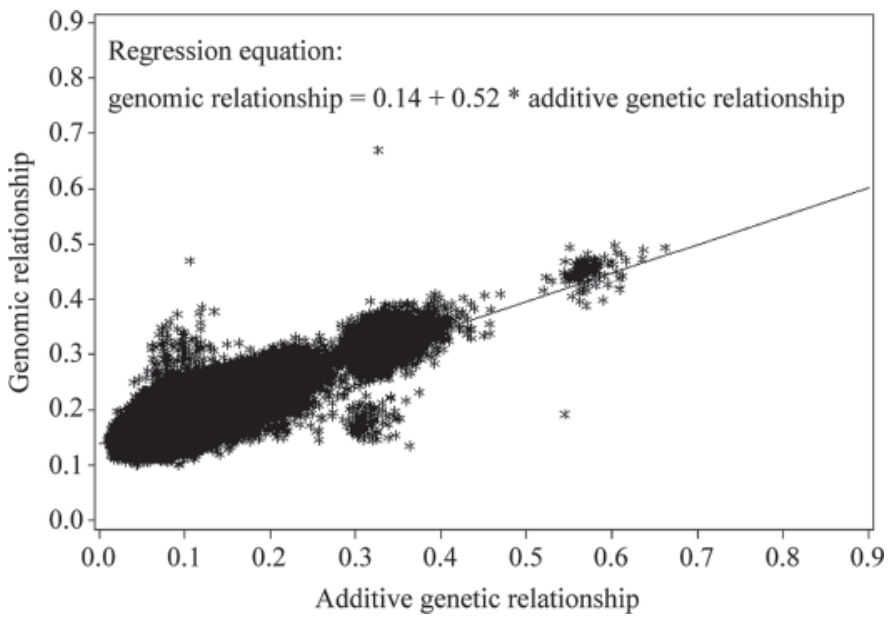

Figure 1. Relationship coefficients estimated from SNP data (genomic relationship) against relationship coefficients estimated from pedigree data (additive genetic relationship).

contributions of genetically favorable single bulls were possible. These findings are in line with those of Kearney et al. (2004) and of König and Simianer (2006) when applying OGC as implemented in GENCONT. In addition, Weigel and Lin (2002) showed that an increase in allowed relationships decreased the number of selected sires. Kearney et al. (2004) associated genetic contributions and index scores for different levels of constraint on inbreeding for selected males, and they found a higher proportion of selected males with a more stringent constraint on rate of inbreeding. Interestingly, more sires were selected for all scenarios when restricting genomic relationships, compared with restrictions on pedigree relationships. For example, at a constraint of $f_{i j}=0.185$ and selection based on Index-PROD, the number of selected sires was 35. In contrast, only 21 sires were selected at the comparable constraint on additive genetic relationship of $\mathrm{a}_{i j}=0.09$ (Figure 4).

When selection was constrained on additive genetic relationships, average Index-PROD and average IndexSCS of selected animals were maximal for an allowed restriction of $a_{i j}=0.15$ (Figure 2 and Figure 3). For constraints on genomic relationships, we observed a continuous increase in genetic gain over the whole range of constraints from $f_{i j}=0.185$ to $f_{i j}=0.24$. The average value of Index-PROD was generally lower when

Table 2. Average coefficients of relationship $( \pm \mathrm{SD})$ for selection candidates

\begin{tabular}{lcc}
\hline $\begin{array}{l}\text { Selection } \\
\text { candidate }\end{array}$ & $\begin{array}{c}\text { Additive genetic } \\
\text { relationship }\end{array}$ & $\begin{array}{c}\text { Genomic } \\
\text { relationship }\end{array}$ \\
\hline 484 bull sires & $0.105 \pm 0.045$ & $0.192 \pm 0.029$ \\
499 bull dams & $0.097 \pm 0.045$ & $0.189 \pm 0.030$ \\
983 bull sires and dams & $0.098 \pm 0.041$ & $0.190 \pm 0.035$ \\
484 bull sires with 499 bull dams & $0.095 \pm 0.035$ & $0.187 \pm 0.025$ \\
\hline
\end{tabular}




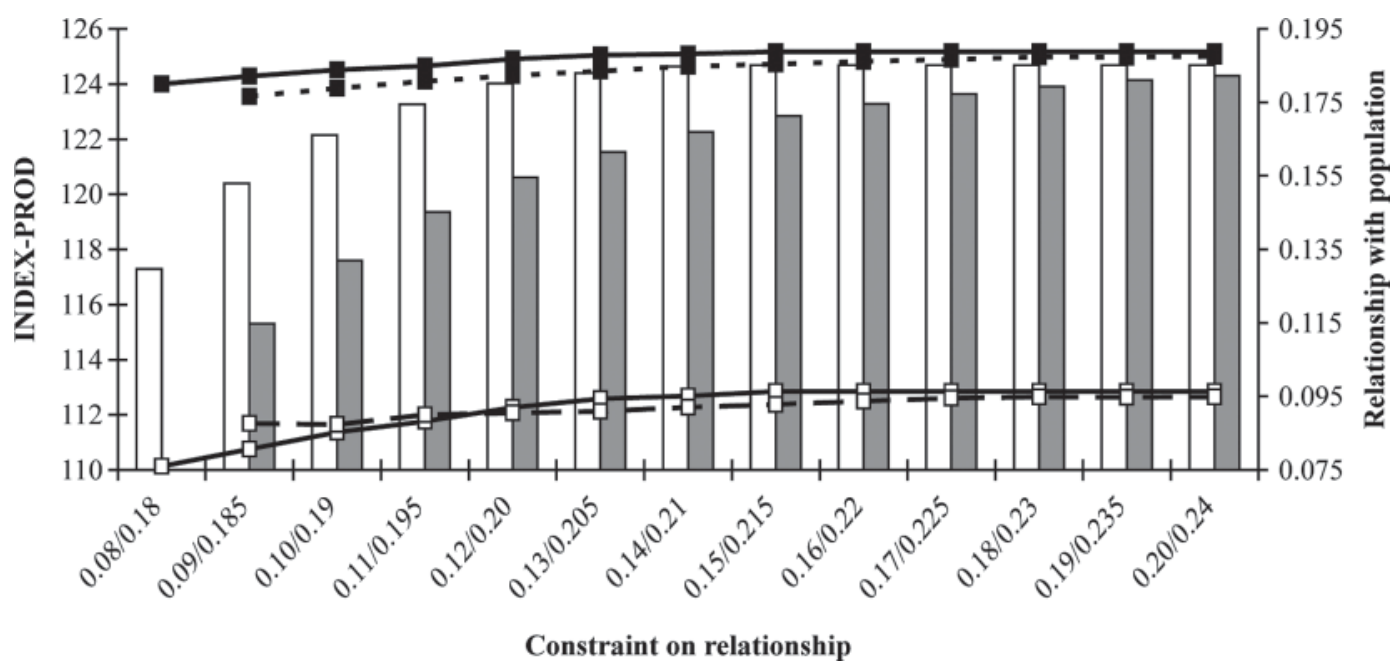

Figure 2. Average production index (Index-PROD) of selected bull sires and bull dams and their average relationship at different constraints for additive genetic and genomic relationships (white bars = average Index-PROD for constraint on additive genetic relationship; gray bars = average Index-PROD for constraint on genomic relationship; solid lines = constraint on additive genetic relationships; dashed lines $=$ constraint on genomic relationships; lines with white squares = realized additive genetic relationships; lines with black squares = realized genomic relationships).

selection was restricted on genomic relationships. This finding is in line with results from a comprehensive simulation study for aquaculture species conducted by Sonesson et al. (2010). In their study, genetic gain was 37 to $60 \%$ higher when constraining the increase in inbreeding based on pedigree data compared with constraints in inbreeding based on genomic data. No optimum solution was found for a constraint of $f_{i j}=$ 0.18 on genomic relationships, when the minimum contribution of an individual sire was set to $2 \%$.

For severe constraints on genomic relationships; that is, in the range from $f_{i j}=0.185$ to $f_{i j}=0.195$, solutions for additive genetic relationships were more relaxed compared with corresponding restrictions of pedigree relationships (Figure 2 and Figure 3; comparison of lines with white squares). When increasing the allowed relationships from $f_{i j}=0.20$ to $f_{i j}=0.24$, solutions for coefficients of additive genetic relationships were higher compared with corresponding restrictions on additive genetic relationships (i.e., altering $a_{i j}=0.12$ to $a_{i j}=$ 0.20). This was found by Sonesson et al. (2010) for all analyzed scenarios. However, differences in solutions for additive genetic relationships were marginal, probably random, but interesting in the observed trend.

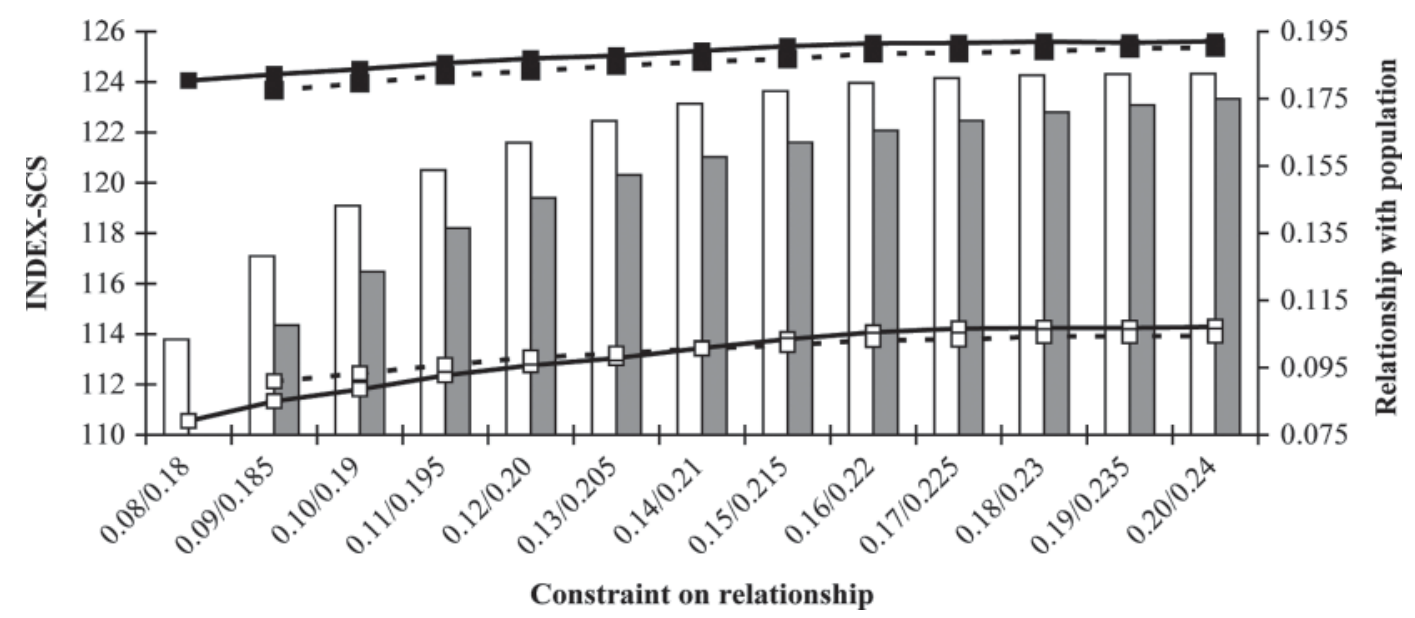

Figure 3. Average SCS index (Index-SCS) of selected bull sires and bull dams and their average relationship at different constraints for additive genetic and genomic relationships (white bars = average Index-SCS for constraint on additive genetic relationship; gray bars $=$ average Index-SCS for constraint on genomic relationship; solid lines = constraint on additive genetic relationships; dashed lines = constraint on genomic relationships; lines with white squares = realized additive genetic relationships; lines with black squares = realized genomic relationships). 


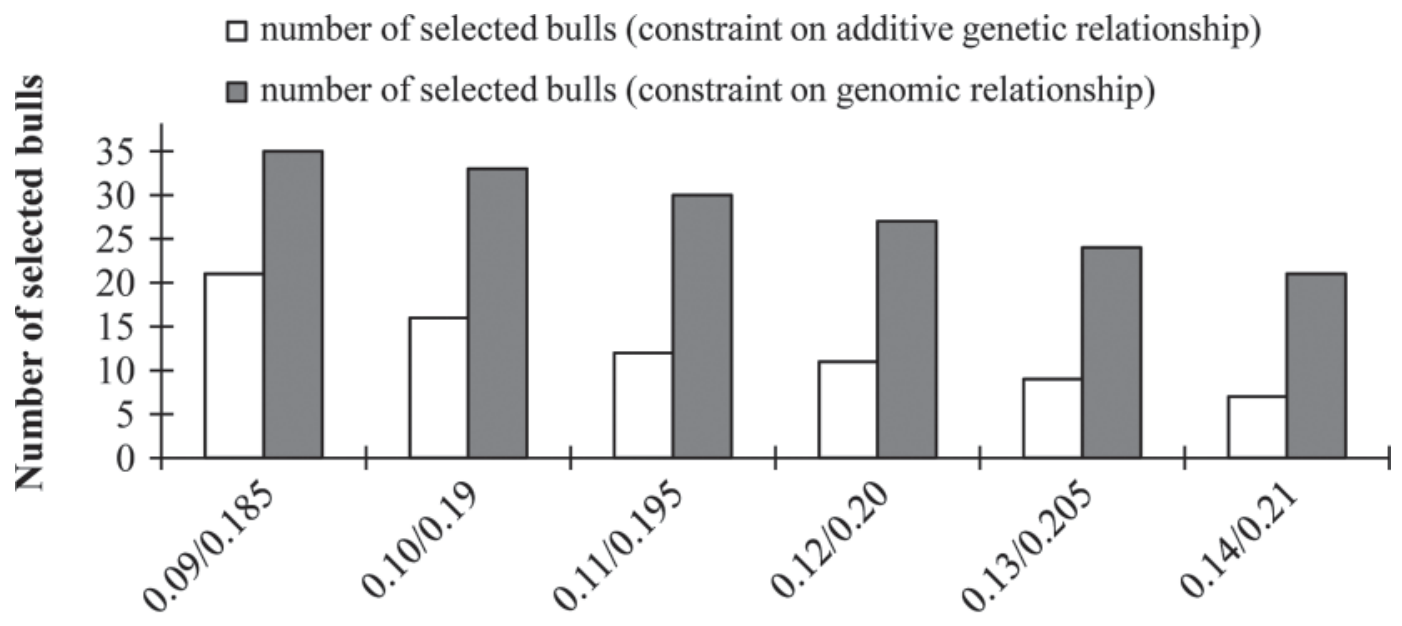

\section{Constraint on relationship}

Figure 4. Number of selected bull sires at different constraints on average additive genetic and genomic relationships (selection criterion: Index-PROD).

According to Sonesson et al. (2010), solutions for realized genomic relationships were generally lower when restricting selection on genomic relationships compared with restrictions on pedigree relationships (Figure 2 and Figure 3, comparison of black squares). As expected, a target-oriented restriction produced more desired results. This desired advantage (i.e., substantially lower solutions for average genomic relationships) decreased with an increase in allowed restrictions. The same trends for solutions in terms of genetic gain and realized relationships were observed for the high heritability trait (Index-PROD, Figure 2) and the low heritability trait (Index-SCS, Figure 3). Although more bulls were selected in the Index-SCS than in the Index-
PROD scenario (comparison of results in Figure 4 and Figure 5), realized relationships were marginally higher for Index-SCS scenarios, perhaps due to the lower SD in Index-SCS for bull sires.

Associations between genetic contributions of selected sires and their indices for 4 different constraints on relationships are depicted in Figure 6 and Figure 7. Differences in genetic contributions among selected sires increased with a relaxing constraint on coefficients of relationships, which is in line with previous studies (e.g., Kearney et al., 2004). In our study and in contrast to results by Kearney et al. (2004) and König and Simianer (2006), selected sires with the highest EBV always had the highest genetic contributions. This was

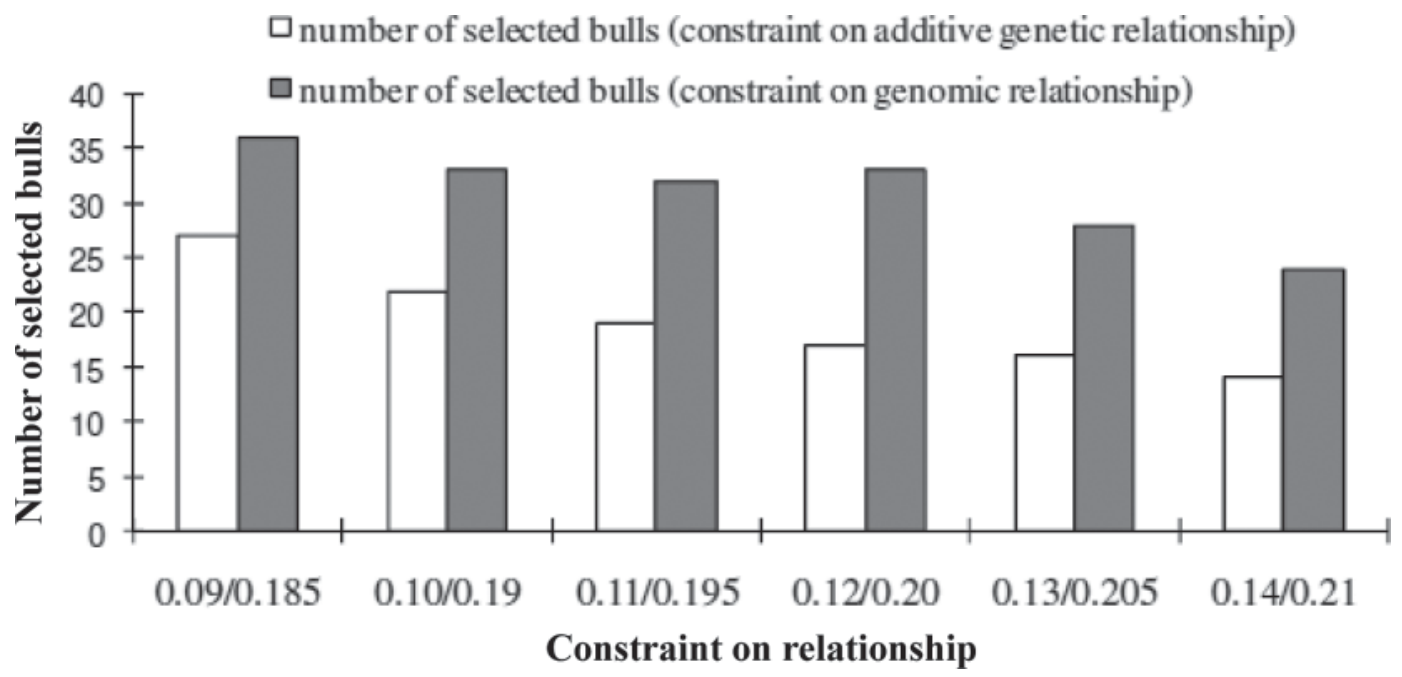

Figure 5. Number of selected bull sires at different constraints on average additive genetic and genomic relationships (selection criterion: Index-SCS). 


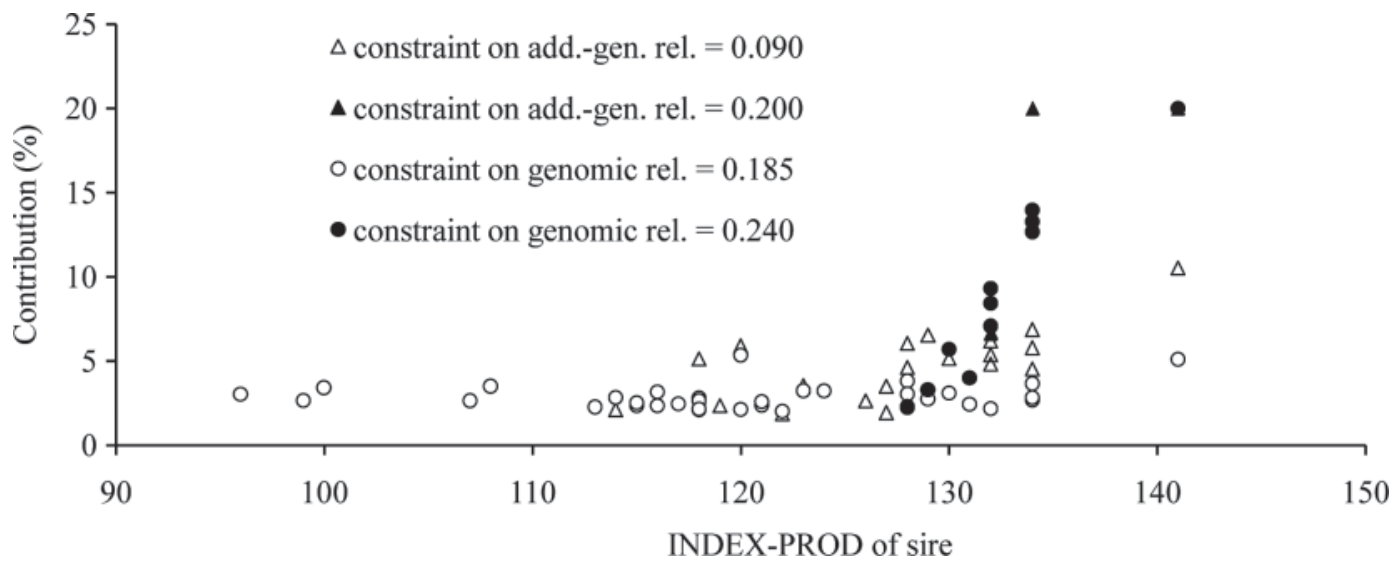

Figure 6. Association between optimized genetic contributions of bull sires and their production index (Index-PROD) for 2 levels of constraint on average additive genetic (add.-gen. rel.) and genomic relationship (genomic rel.).

probably a coincidence because those animals had relatively low coefficients of relationship. For example, the 4 best sires for Index-PROD had an average additive genetic relationship with the population of $a_{i j}=0.089$, and a genomic relationship of $f_{i j}=0.183$. Generally, a wider range for Index-PROD (Figure 6) and Index-SCS (Figure 7) of selected sires was found when constraining genomic relationships.

\section{Specific Mating Designs}

Average relationships of mating partners when combining results from the SDP algorithm with simulated annealing are shown in Figure 8 and Figure 9 for IndexPROD and Index-SCS, respectively. Following these results, a further reduction of coefficients for genomic and pedigree relationships is possible only when focusing on the minimization of relationships or inbreeding coefficients of individuals in the following generation by using an annealing algorithm as proposed by Sones- son and Meuwissen (2000). For example, with selection based on Index-PROD and restricting the maximal additive genetic relationship to a value of $a_{i j}=0.09$, all possible sire-dam pairs suggested by the SDP algorithm had an average additive genetic relationship of $a_{i j}=0.068$ and an average genomic relationship of $f_{i j}$ $=0.179$. Matings proposed by the annealing algorithm had an average additive genetic relationship of $a_{i j}=$ 0.048 and an average genomic relationship of $f_{i j}=0.164$. A comparable scenario using SDP with a constraint on genomic relationship at $f_{i j}=0.185$ led to an average additive genetic relationship of all possible sire-dam pairs of $a_{i j}=0.078$, and an average genomic relationship of $f_{i j}=0.165$. The average additive genetic relationship was $a_{i j}=0.06$, and average genomic relationship was $f_{i j}=0.135$ when subsequently applying the simulated annealing algorithm.

The results from the simulated annealing algorithm were also in agreement with calculations conducted in another region of Germany for Holstein dairy cattle

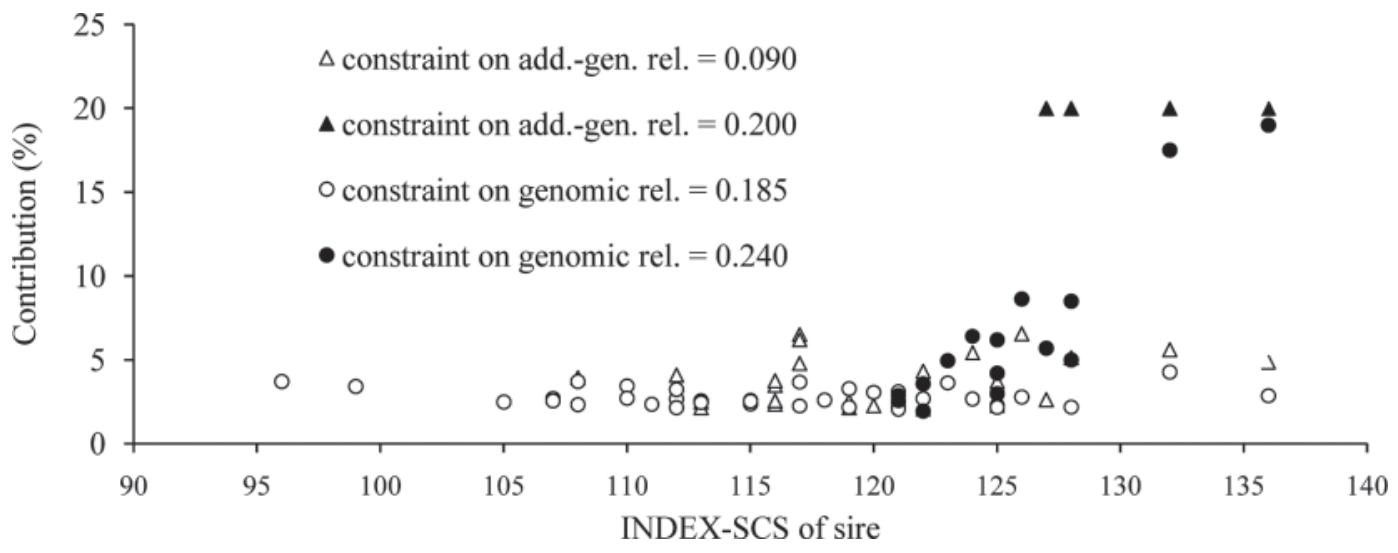

Figure 7. Association between optimized genetic contributions of bull sires and their somatic cell score index (Index-SCS) for 2 levels of constraint on average additive genetic (add.-gen. rel.) and genomic relationship (genomic rel.). 


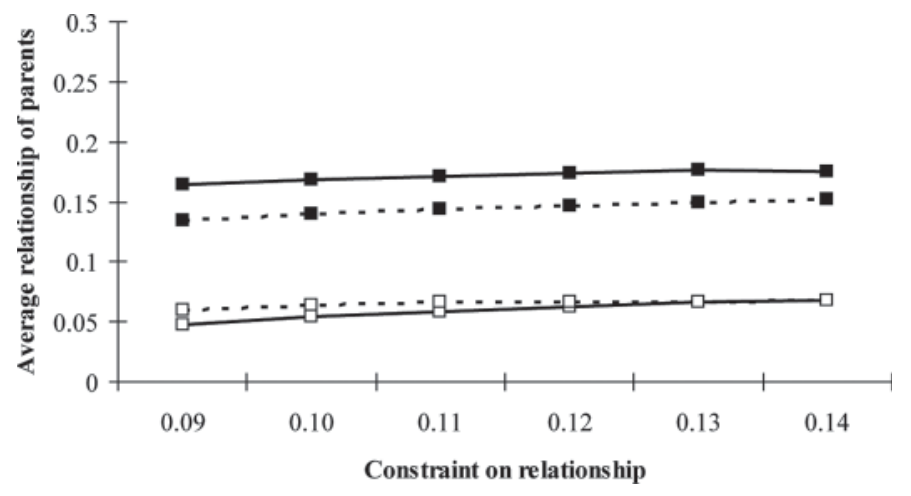

Figure 8. Average relationships of parents of the next generation when specifying matings with an annealing algorithm at different constraints for average additive genetic and genomic relationships (selection criterion: Index-PROD; solid lines = constraint on additive genetic relationships; dashed lines $=$ constraint on genomic relationships lines with white squares $=$ realized additive genetic relationships; lines with black squares $=$ realized genomic relationships).

(König and Simianer, 2006). As one specific example from this study, 21 selected bulls and 30 selected cows were mated at random. The expected inbreeding coefficient for their progeny was $1.38 \%$. In contrast, the simulated annealing algorithm suggested a mating plan with an average inbreeding coefficient in the next generation of only $0.46 \%$.

Again, the mating design from the simulated annealing algorithm can be considered a strategy for avoiding or minimizing inbreeding in the short term. Such a strategy could make sense for species or breeding plans characterized by long generation intervals, especially in dairy cattle or horse breeding programs (Niemann et al., 2009). For species with short generation intervals (e.g., pig or poultry breeding), the advantage from

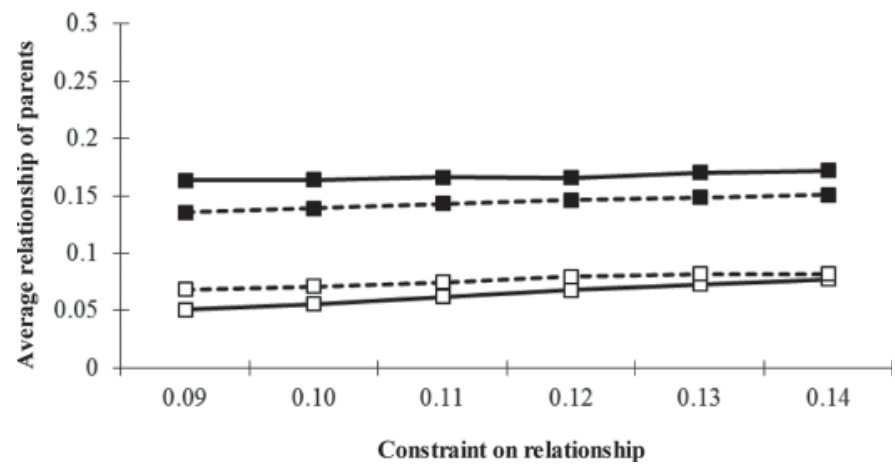

Figure 9. Average relationships of parents of the next generation when specifying matings with an annealing algorithm at different constraints for average additive genetic and genomic relationships (selection criterion: Index-SCS; solid lines = constraint on additive genetic relationships; dashed lines $=$ constraint on genomic relationships; lines with white squares $=$ realized additive genetic relationships; lines with black squares $=$ realized genomic relationships). minimizing inbreeding in the short-term will erode very quickly (König et al., 2010). But competitive future genomic dairy cattle breeding programs will also be characterized by a substantial shortening in generation intervals, especially on the bull sire and cow sire pathway of selection (Schaeffer, 2006). From this point of view, the general OGC concept without the application of specific mating plans should be the major motivation.

\section{General Perspective}

Daetwyler et al. (2007) pointed out that genomic selection would lead to lower inbreeding than traditional selection schemes because the accuracies of estimated Mendelian sampling terms will be higher. This allows a higher differentiation within families and a lower probability of co-selection of sibs. From the practical point of view (Hayes et al., 2009; König et al., 2009), a shorter generation interval in combination with high accuracies of genomic breeding values for young sires may lead to higher rates of inbreeding. Therefore, methods for optimizing long-term genetic contributions as presented in this study are of major importance to avoid a future increase in inbreeding. Selection on bull sires and bull dams, as well as selection of cow sires, is controlled by breeding organizations. Hence, the OGC concept as an additional "controlling instrument" could easily be implemented in this step of selection (König et al., 2007).

Most of the trends in terms of genetic gain or realized relationships in our study were similar when comparing results from restrictions on pedigree or on genomic relationships, but minor differences could have practical importance. Consequently, the more sophisticated and innovative approach is based on marker data and should be applied for scientific objectives, as suggested by Pimentel et al. (2011b).

We applied the OGC concept for the selection of bull sires and bull dams within a framework of a genomic breeding program as suggested by Schaeffer (2006), and by König et al. (2009). The genomic era further allows for a change from the 4-pathway selection strategy in dairy cattle, as introduced by Rendel and Robertson (1950), to a 2-pathway selection strategy (König and Swalve, 2009). Such a strategy implies that a large number of male calves, all being selection candidates for AI, are genotyped. This would erode the traditional concept of mating designs and selection strategies for bull sires and bull dams. Hence, further improvements for maximizing genetic gain and minimizing relationships should focus on SNP patterns of male calves; for example, the construction of a heterozygosity index. Our approach should be considered as a breeding tool 
in the transitional phase from the conventional toward the genomic era. However, we also assume that some form of preselection in terms of elite matings may still exist, and already at this early step of selection, minimizing relationships will contribute to ensure long-term selection response. Without preselection, the proposed method might be challenging for extremely large data sets but, as stated by Sonesson et al. (2010), using genomic relationships will result in a more precise control of the genomic inbreeding.

\section{CONCLUSIONS}

Due to the continuous increase of inbreeding, the accumulation of defect genes, and the risk of inbreeding depressions, Holstein dairy cattle breeding programs should consider aspects that both maximize genetic gain and minimize long-term genetic relationships. In contrast to previous applications using GENCONT software and pedigree-based relationships, we focused on SDP and relationships constructed from SNP data. For moderately and lowly heritable traits, our approach can identify those mating partners that ensure maximum genetic gain at specific constraints on maximum relationship. Our approach has been suggested to identify elite matings between preselected bull sires and bull dams using SNP data, but the flexibility of this concept (i.e., the combination of pedigree-based and marker data) allows an application for a broad variety of aspects to maintain genetic diversity (e.g., Wang, 2001).

\section{ACKNOWLEDGMENTS}

The authors appreciate funding from the Lower Saxon Ministry of Food, Agriculture, Consumer Protection and Regional Development. Parts of this study were conducted within the projects FUGATO-plus brain and FUGATO-plus GenoTrack, which were financially supported by the German Ministry of Education and Research. Furthermore, assistance from Ricardo PongWong [Roslin Institute and R(D)SVS, University of Edinburgh, Roslin, Midlothian, UK] during the configuration of the semi-definite programming algorithm is kindly appreciated.

\section{REFERENCES}

Daetwyler, H. D., B. Villanueva, P. Bijma, and J. A. Woolliams. 2007. Inbreeding in genome-wide selection. J. Anim. Breed. Genet. 124:369-376.

Eding, H., and T. H. E. Meuwissen. 2001. Marker-based estimates of between and within population kinships for the conservation of genetic diversity. J. Anim. Breed. Genet. 118:141-159.
Emik, L. O., and C. R. Terrill. 1949. Systematic procedures for calculating inbreeding coefficients. J. Hered. 40:51-55.

Fujisawa, K., M. Kojima, K. Nakata, and M. Yamashita. 2002. SDPA (SemiDefinite Programming Algorithm) user's manual, version 6.00. Research Reports on Mathematical and Computer Sciences, Series B: Operations Research. Tokyo Institute of Technology, Tokyo, Japan.

Gengler, N., P. Mayeres, and M. Szydlowski. 2007. A simple method to approximate gene content in large pedigree populations: Application to the myostatin gene in dual-purpose Belgian Blue cattle. Animal 1:21-28.

Hayes, B. J., P. J. Bowman, A. J. Chamberlain, and M. E. Goddard. 2009. Invited review: Genomic selection in dairy cattle: Progress and challenges. J. Dairy Sci. 92:433-443.

Kearney, J. F., E. Wall, B. Villanueva, and M. P. Coffey. 2004. Inbreeding trends and application of optimized selection in the UK Holstein population. J. Dairy Sci. 87:3503-3509.

König, S., S. Lessner, and H. Simianer. 2007. Application of controlling instruments for improvements in cow sire selection. J. Dairy Sci. 90:1967-1980.

König, S., and H. Simianer. 2006. Approaches to the management of inbreeding and relationship in the German Holstein dairy cattle population. Livest. Sci. 103:40-53.

König, S., H. Simianer, and A. Willam. 2009. Economic evaluation of genomic breeding programs. J. Dairy Sci. 92:382-391.

König, S., and H. H. Swalve. 2009. Application of selection index calculations to determine selection strategies in genomic breeding programs. J. Dairy Sci. 92:5292-5303.

König, S., F. Tsehay, F. Sitzenstock, U. U. v. Borstel, M. Schmutz, R. Preisinger, and H. Simianer. 2010. Evaluation of inbreeding in laying hens by applying optimum genetic contribution and gene flow theory. Poult. Sci. 89:658-667.

Meuwissen, T. H. E. 1997. Maximizing the response of selection with a predefined rate of inbreeding. J. Anim. Sci. 75:934-940.

Meuwissen, T. H. E. 2002. GENCONT: An operational tool for controlling inbreeding in selection and conservation schemes. Proc. 7th World Congr. Genet. Appl. Livest. Prod., Montpellier, France. CD-Rom Commun. no. 28:20.

Miglior, F. 2000. Impact of inbreeding-Managing a declining Holstein pool. Pages 108-113 in Proc. 10th World Holstein Friesian Conf., Sydney, Australia. World Holstein Friesian Federation, Rickmansworth, UK.

Mrode, R., J. F. Kearney, S. Biffani, M. Coffey, and F. Canavesi. 2009. Short communication: Genetic relationships between the Holstein cow populations of three European dairy countries. J. Dairy Sci. 92:5760-5764.

Niemann, B., S. König, and E. Bruns. 2009. Maximizing genetic gain by restricting inbreeding in Hanoveranian breeding programme for show jumpers. Zuchtungskunde 81:51-57.

Pimentel, E. C. G., S. Bauersachs, M. Tietze, H. Simianer, J. Tetens, G. Thaller, F. Reinhardt, E. Wolf, and S. König. 2011b. Exploration of relationships between production and fertility traits in dairy cattle via association studies of SNPs within candidate genes derived by expression profiling. Anim. Genet. 42:251-262.

Pimentel, E. C. G., M. Erbe, S. König, and H. Simianer. 2011a. Genome partitioning of genetic variation for milk production and composition traits in Holstein cattle. Front. Livest. Genomics 2:19. doi:10.3389/fgene.2011.00019.

Pong-Wong, R., and J. A. Woolliams. 2007. Optimisation of contribution of candidate parents to maximise genetic gain and restricting inbreeding using semidefinite programming. Genet. Sel. Evol. $39: 3-25$

Press, W. H., B. P. Flannery, S. A. Teukolsky, and W. T. Vetterling. 1989. Minimization or maximization of functions, Pages 274-334 in Numerical Recipes-The Art of Scientific Computing. University of Cambridge Press, Cambridge, UK.

Rendel, J. M., and A. Robertson. 1950. Estimation of genetic gain in milk yield by selection in a closed herd of dairy cattle. J. Genet. $50: 1-8$. 
Sanders, K., J. Bennewitz, and E. Kalm. 2006. Wrong and missing sire information affects genetic gain in the Angeln dairy cattle population. J. Dairy Sci. 89:315-321.

Schaeffer, L. R. 2006. Strategy for applying genome-wide selection in dairy cattle. J. Anim. Breed. Genet. 123:218-223.

Schork, N. J. 2001. Genome partitioning and whole-genome analysis. Adv. Genet. 42:299-322.

Sonesson, A. K., and T. H. Meuwissen. 2000. Mating schemes for optimum contribution selection with constrained rates of inbreeding. Genet. Sel. Evol. 32:231-248.

Sonesson, A. K., J. A. Woolliams, and T. H. E. Meuwissen. 2010. Maximising genetic gain whilst controlling rates of genomic inbreeding using genomic optimum contribution selection. Abstract no. 892 in Proc. 9th World Congr. Genet. Appl. Livest. Prod., Leipzig, Germany. German Society for Animal Science, Leipzig, Germany.

Thompson, J. R., R. W. Everett, and C. W. Wolfe. 2000. Effects of inbreeding on production and survival in Jerseys. J. Dairy Sci. $83: 2131-2138$

VanRaden, P. M. 2005. Inbreeding adjustments and effect on genetic trend estimates. Interbull Bull. 33:81-84.

VanRaden, P. M., C. P. Van Tassel, G. R. Wiggans, T. S. Sonstegard, R. D. Schnabel, J. F. Taylor, and F. S. Schenkel. 2009. Invited review: Reliability of genomic predictions for North American Holstein bulls. J. Dairy Sci. 92:16-24.

Wang, J. 2001. Optimal marker-assisted selection to increase the effective size of small populations. Genetics 157:867-874.

Weigel, K. A., and S. W. Lin. 2002. Controlling inbreeding by constraining the average relationship between parents of young bulls entering AI progeny test programs. J. Dairy Sci. 85:2376-2383.

Weller, J. I., E. Feldmesser, M. Golik, I. Tager-Cohen, R. Domochowsky, O. Alus, E. Ezra, and M. Ron. 2004. Factors affecting incorrect paternity assignment in the Israeli Holstein population. J. Dairy Sci. 87:2627-2640.

Wensch-Dorendorf, M., T. Yin, H.H. Swalve, and S. König. 2011. Optimal strategies for the use of genomic selection in dairy cattle breeding programs. J. Dairy Sci. 94:4140-4151. doi:10.3168/ jds.2010-4101.

Wiggans, G. R., P. M. Van Raden, and J. Zuurbier. 1995. Calculation and use of inbreeding coefficients for genetic evaluation of United States dairy cattle. J. Dairy Sci. 78:1584-1590.

Wright, S. 1922. Coefficients of inbreeding and relationship. Am. Nat. $56: 330-338$ 